\title{
MÉTHODE GÉNÉRALE DE CALCUL DES CHAMBRES D'ÉQUILIBRE ALIMENTÉES PAR 2 CANAUX D'AMENÉE.
}

par V. GOUTKIN, Ingénieur I.E.G. et E.I.H.

Dans le "GÉNIE CIVIL » des $1^{\text {er }}$ et 15 avril 1943, Monsieur L. ESCANDE a exposé une méthode de calcul des chambres d'équilibre dans le cas où l'aménagement hydroélectrique comporte 2 (ou plusieurs) canaux d'amenée. Cette méthode qui introduit la notion nouvelle de canal équivalent et permet une généralisation à tous les systèmes possibles, présente cependant à la base une hypothèse restrictive, les lacs d'alimentation devant nécessairement être à la même cote statique.

Nous avons donc repris l'étude de cette question en envisageant le cas général, souvent réalisé en pratique, où il existe une différence A entre les cotes des plans d'eau dans les 2 lacs, ces cotes étant supposées constantes pendant la durée des oscillations dans la chambre.

Nous bornerons le développement qui vo suivre au cas des 2 canaux d'amenée en $V$ (chambre d'équilibre située au confluent des 2 canaux), mais son application au cas de canaux

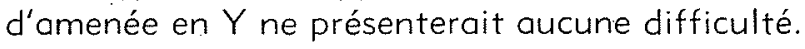

\section{CHAPITRE}

\section{PRINCIPES DE CALCUL}

Nous désignerons par:

$f_{1}, f_{2}$ les sections respectives d'écoulement dans les 2 canaux d'amenée.

$L_{1}, L_{2}$ les longueurs de ces canaux.

$P_{1}=K_{2} q^{2}{ }_{1}, P_{2}=K_{2} q_{2}^{2}$ les pertes de charge pour les débits $q_{1}=f_{1} w_{1}, q_{2}=f_{2} w_{2}$ comptés positivement vers la chambre.

$\checkmark$ la vitesse d'écoulement dans la chambre, comptée positivement vers le haut.

$Z_{1}, Z_{2}$ les cotes du plan d'eau dans la chambre rapportée aux niveaux respectifs $O$ et $-A$ des 2 lacs.
Q le débit des conduites forcées à un instant quelconque $\mathrm{t}$.

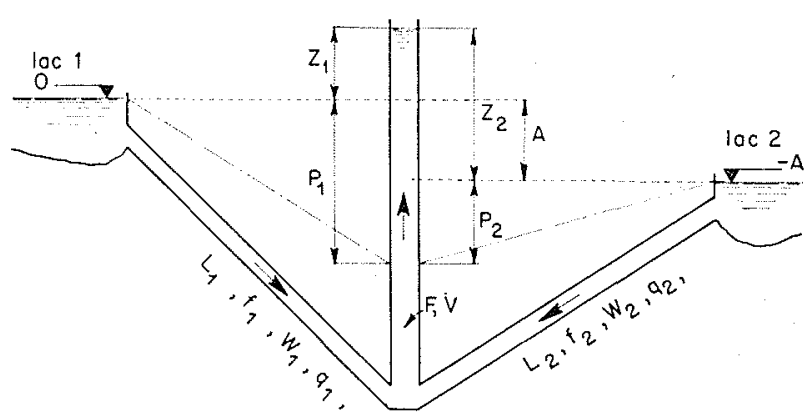

fig. 1

L'équation de continuité s'écrit immédiatement (fig. 1) :

$$
\mathrm{q}_{1}+\mathrm{q}_{2}=\mathrm{f}_{1} \mathrm{w}_{1}+\mathrm{f}_{2} \mathrm{w}_{2}=\mathrm{FV}+\mathrm{Q}
$$

ou en dérivant par rapport au temps:

$$
f_{1} \frac{d w_{1}}{d t}+f_{2} \frac{d w_{2}}{d t}=\frac{F d V}{d t}+\frac{d Q}{d t}
$$

Appliquons maintenant le théorème des quantités de mouvement à la masse d'eau contenue dans chacun des 2 canaux (1) et (2), en négligeant la variation de quantité de mouvement de l'eau à l'intérieur de la chambre d'équilibre.

Nous obtenons les relations suivantes:

$$
\begin{aligned}
& \frac{L_{1}}{g} \frac{d W_{1}}{d t}+Z_{1}+i_{1} P_{1}=0 \\
& \frac{L_{2}}{g} \frac{d W_{2}}{d t}+Z_{2}+\Sigma_{2} P_{2}=0
\end{aligned}
$$

dans lesquelles $\Sigma_{1}$ et $\Sigma_{2}$ égaux d̀ +1 , ont respectivement les signes de $a_{1}$ et $q_{2}$ (II n'y a du reste lieu d'introduire ces facteurs de signe $\varepsilon_{1}$ et $\varepsilon_{2}$ que lorsque la perte de charge est considérée comme étant une fonction paire du débit). 
Notre méthode de calcul est basée sur l'approximation consistant à admettre que la relation entre pertes de charge:

$$
\varepsilon_{1} P_{1}=\Sigma_{2} P_{2}+A
$$

qui existe en régime permanent, demeure sensiblement valable pendant les oscillations.

Comme d'autre part on a constamment:

$$
z_{2}=z_{1}+A
$$

les relations (5) et (6) permettent d'écrire:

$$
Z_{1}+\hat{\varepsilon}_{1} P_{1}=Z_{2}+\Sigma_{2} P_{2}
$$

Il est alors possible, comme nous allons le voir ci-après, de résoudre le problème sans recourir à la méthode par différences finies, également exposée par $M$. ESCANDE, mathématiquement exacte, mais d'une application beaucoup plus longue.

\section{CHAPITRE $\mid$ I}

\section{ÉTUDE DE LA FERMETURE COMPLÈTE INSTANTANÉE A PARTIR DU DÉBIT MAXIMUM $Q$, DES TURBINES}

Le débit des conduites étant égal à 0 dès le début du phénomène, l'équation de continuité (2) devient:

$$
f_{1} \frac{d W_{1}}{d t}+f_{2} \frac{d W_{2}}{d t}=F \frac{d V}{d t}
$$

Des équations (3) et (4) tirons les valeurs de $\frac{d W_{1}}{d t}$ et $\frac{d W_{2}}{d t}$ que nous porterons dans ( $\left.{ }^{\mathrm{bis}}\right)$.

II vient successivement :

$$
\begin{aligned}
& \frac{d W_{1}}{d t}=\frac{-g}{L_{1}}\left(Z_{1}+\varepsilon_{1} P_{1}\right) \\
& \frac{d W_{2}}{d t}=-\frac{-g}{L_{2}}\left(Z_{2}+\Sigma_{2} P_{2}\right)
\end{aligned}
$$

et en vertu de l'égalité :

$$
\frac{d W_{2}}{d t}=-\frac{g}{L_{2}}\left(Z_{1}+\varepsilon_{1} P_{1}\right)
$$

et enfin :

$$
F \frac{d V}{d t}+g\left(\frac{t_{1}}{L_{1}}+\frac{t_{2}}{L_{2}}\right) Z+g\left(\frac{t_{1}}{L_{1}}+\frac{t_{2}}{L_{2}}\right) \epsilon_{1} P_{1}=0
$$

$$
\begin{aligned}
& \text { Posons: } \\
& \qquad \frac{f}{L}=\frac{f_{1}}{L_{1}}+\frac{f_{2}}{L_{2}}
\end{aligned}
$$

L'équation précédente s'écrit alors :

$$
\frac{L F}{g f} \frac{d V}{d t}+z_{1}+\varepsilon_{1} P_{1}=0
$$

ou encore:

$$
\frac{L F}{g f} \vee \frac{d V}{d z}+Z_{1}+\varepsilon_{1} P_{1}=0
$$

L'expression (9) n'est autre que l'équation de mouvement obtenue avec un canal fictif équivalent, défini par l'égalité (8) et dans lequel ia perte de charge serait constamment égale à celle $\mathrm{P}_{1}$ créée par le canal 1 .

La surélévation $Z_{1 m}$ dans la chambre sera évidemment comptée à partir du niveau du lac 1.

De même, on pourrait écrire:

$$
\frac{L F}{g f} V \frac{d V}{d Z}+Z_{2}+\varepsilon_{2} P_{22}=0
$$

La surélévation maximum $Z_{p m}$ dans la chambre, comptée à partir du niveau statique (- A) du lac 2, serait celle obtenue avec un canal fictif équivalent, toujours défini par la relation (8) et où la perte de charge serait constamment égale à $P_{\underline{n}}$.

\section{INTÉGRATION GRAPHIQUE}

\section{1 - Equation différentielle :}

L'équation (9) (ou 9. bis) n'est pas intégrale analytiquement, mais elle se prête, en revanche, à la méthode de résolution graphique.

En effet, posons pour simplifier :

$$
C=\frac{g^{f}}{F L}
$$

L'équation (9) s'écrit :

$$
\frac{d V}{d Z}=-c \frac{Z_{1}+P_{1}}{V}
$$

que nous intégrerons sous cette forme, après avoir examiné la perte de charge $P_{1}$ en fonction de $\mathrm{V}$. 


\section{2 - Expression de la perte de charge :}

L'égalité (5) S'écrit identiquement:

$$
\xi_{1} K_{1} q_{1}^{2}=\approx_{2} K_{2} q_{2}{ }^{2}+A
$$

et puisque $: q_{1}+q_{2}=F V$

$$
\varepsilon_{1} K_{1} q_{1}^{2}=\varepsilon_{2} K_{2}\left(F V-q_{1}\right)^{2}+A
$$

La résolution de cette dernière expression conduit à :

$$
q_{1}=\frac{-\epsilon_{2} K_{2} F V+\sqrt{\epsilon_{1} \epsilon_{2} K_{1} K_{2} F^{2} V^{2}+A\left(\epsilon_{1} K_{1}-\epsilon_{2} K_{2}\right)}}{\epsilon_{1} K_{1}-\epsilon_{2} K_{2}}
$$

D'où l'on tire :

$$
P_{1}=\frac{\left.\epsilon_{1} \epsilon_{2} K_{1} K_{2}\left(\epsilon_{1} K_{1}+\epsilon_{2} K_{2}\right) F^{2} V^{2}+\epsilon_{1} K_{1}\left(\epsilon_{1} K_{1}-\epsilon_{2} K_{2}\right) A-2 \epsilon_{1} \epsilon_{2} K_{1} K_{2} F V \sqrt{\left(\epsilon_{1} \epsilon_{2} K_{1} K_{2} F^{2} V^{2}+A\left(\epsilon_{1} K_{1}-\epsilon_{2} K_{2}\right.\right.}\right)}{\left(\epsilon_{1} K_{1}-\epsilon_{2} K_{2}\right)^{2}}
$$

On peut dès lors, tracer la courbe $P_{1}=0(V)$, en remarquant que pendant le début de la $l^{\text {ro }}$ montée, on a :

$$
\mathrm{q}_{1}>0 \quad \mathrm{q}_{2}>0 \quad \varepsilon_{1}=+1 \quad \varepsilon_{2}=+1
$$

jusqu'au moment où commence le remplissage du lac 2 par le lac 1, c'est-à-dire jusqu'à ce que:

$$
P_{1}=A=K_{1} F^{2} V^{2}, a_{1}=F V, a_{2}=0, V=\frac{1}{F} \sqrt{\frac{A}{K_{1}}}
$$

Ensuite $\mathrm{q}_{2}$ et $\varepsilon_{2}$ deviennent négatifs.

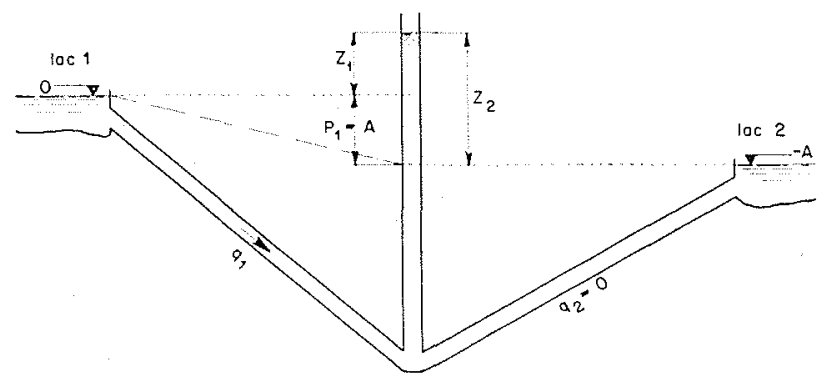

fig. 2

\section{3 - Construction du Graphique :}

Les relations précédentes (11 et 13) permettent de construire graphiquement, par le procédé habituel des normales, la courbe $Z_{1}=f(V)$ et de déterminer en particulier la cote maximum $Z_{1 m}$ atteinte par l'oscillation dans la chambre, à la fin de la $1^{\text {ra }}$ montée (fig. 3).

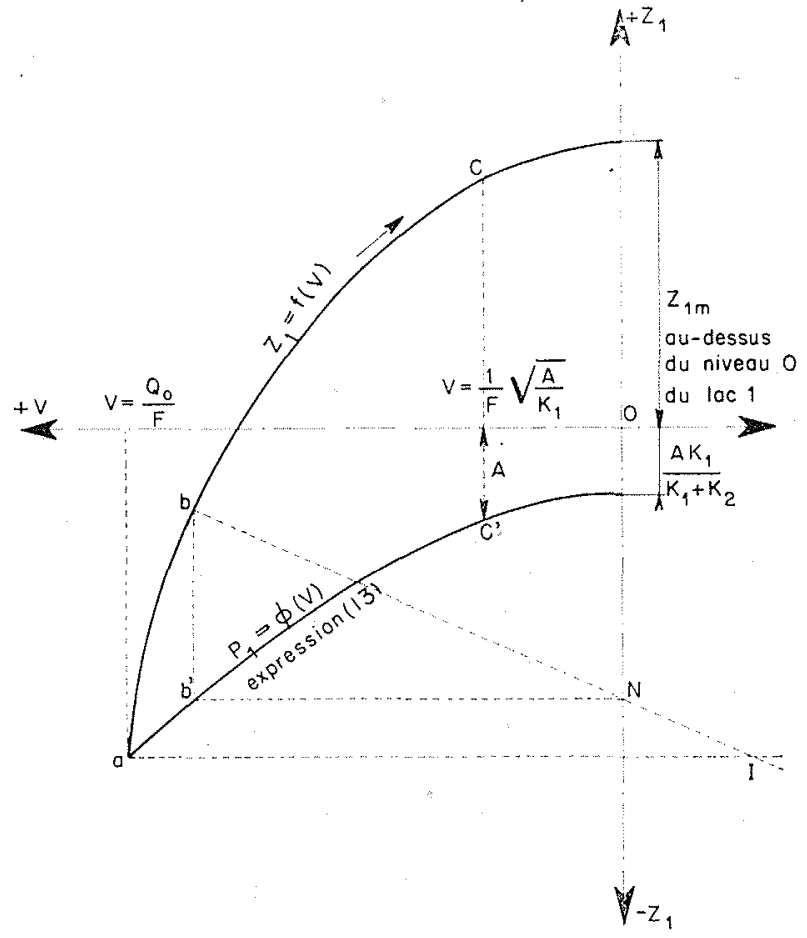

fig. 3

Les conditions initiales de mouvement sont :

$V=\frac{Q_{0}}{F} \quad Z_{1}=-P_{1} \quad \frac{d V}{d Z}=0$

et le rayon de courbure :

$$
P_{0}=-\frac{Q_{0}}{g} \frac{1}{\frac{i_{1}}{L_{1}}+\frac{i_{2}}{L_{2}}}
$$


Le rayon de courbure présente une discontinuité au point (c) d'abscisse

$$
V=\frac{1}{F} \frac{A}{K_{1}}
$$

\section{4 - Variables sans dimensions :}

Il y a toujours intérêt, poúr la rapidité de la construction, à utiliser les valeurs relatives.

Ainsi, en posant :

$$
v=\frac{V}{v_{0}} \quad z_{1}=\frac{Z_{1}}{Z_{*}} \quad p_{1}=\frac{P_{1}}{Z_{*}}
$$

avec

$$
z_{*}=v_{0} \sqrt{\frac{F}{g} \frac{1}{\frac{f_{1}}{L_{1}}+\frac{f_{2}}{L_{2}}}}
$$

l'équation (11) devient :

$$
\frac{d v}{d z}=-\frac{z_{1} P_{1}}{v}
$$

et les conditions initiales de mouvement :

$v=1 \quad z_{1}=-p_{1} \quad \frac{d v}{d z}=0 \quad p_{0}=-1$

En raison de la multiplicité des données, la méthode ne peut être généralisée, en ce sens qu'elle ne permet pas l'établissement d'un abaque classificatif.

Pour chaque cas étudié, il faut done faire intervenir la solution graphique.

\section{5 - Cas particulier :}

\section{Les 2 lacs sont au même niveau $(A=0)$}

En vertu de I'hypothèse de base (4) on a immédiatement :

$$
\mathrm{P}_{1}=\mathrm{P}_{2}=\mathrm{P}
$$

Enfaisant $A=0$ dans I'expression (13) de $P_{1}$ il résulte après quelques simplifications:

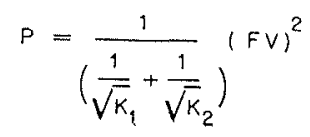

soit :

$$
P=K F^{2} V^{2}
$$

On retrouve ainsi la formule proposée par Monsieur ESCANDE.

Exprimons la perte de charge $P$ en fonction de $P_{0}$ correspondant au début permanent initial :

$$
P=P_{0}\left(\frac{F V}{F V_{0}}\right)^{2}=P_{0} V^{2}
$$

et en valeurs relatives:

$$
\mathrm{P}=\mathrm{P}_{0} \mathrm{v}^{2}
$$

L'équation différentielle de mouvement devient alors :

$$
\frac{d v}{d z}=-\frac{z+p_{0} v^{2}}{v}
$$

La valeur maximum relative $z_{n}$ ne dépendant que de celle de $p_{0,}$, il est possible dans ce cos, d'utiliser l'abaque classificatif de fermeture figurant dans l'ouvrage de MM. CALAME et GADEN «Théorie des Chambres d'équilibre ».

CHAPITRE $\|$

\section{ÉTUDE D'UNE OUVERTURE COMPLÈTE INSTANTANÉE DE 0 à $Q_{1}$}

L'équation de mouvement demeure :

$$
\frac{d V}{d Z}=-c \frac{Z_{1}+P_{1}}{V}
$$

Vis-à-vis des relations précédentes, seule change l'expression de la perte de charge $P_{1}$.

En effet, en considérant l'égalité :

$$
\varepsilon_{1} K_{1} q_{1}^{2}=\varepsilon_{2} K_{2} q_{2}{ }^{2}+A
$$

et remarquant que cette fois :

$$
\mathrm{q}_{1}+\mathrm{q}_{2}=\mathrm{FV}+\mathrm{Q}_{0}
$$


on obtient :

$$
P_{1}=\frac{\epsilon_{1} \epsilon_{2} K_{1} K_{2}\left(\epsilon_{1} K_{1}+\epsilon_{2} K_{2}\right)\left(F V+Q_{0}\right)^{2}+\epsilon_{1} K_{1}\left(\epsilon_{1} K_{1}-\epsilon_{2} K_{2}\right) A-2 \epsilon_{1} \epsilon_{2} K_{1} K_{2}\left(F V+Q_{0}\right) \sqrt{\epsilon_{1} \epsilon_{2} K_{1} K_{2}\left(F V+Q_{0}\right)^{2}+A\left(\epsilon_{1} K_{1}-\epsilon_{2} K_{2}\right)}}{\left(\epsilon_{1} K_{1}-\epsilon_{2} K_{2}\right)^{2}}
$$

Au début de la descente, on a :

$\mathrm{q}_{1}>0 \cdot \mathrm{q}_{2}<0 \quad \dot{\Sigma}_{1}=+1 \quad \dot{\varepsilon}_{2}=-1$ jusqu'au moment où cesse le remplissage du lac 2 par le lac 1, c'est-à-dire jusqu'à ce que:

$P_{1}=A=K_{1} q_{1}{ }^{2} \quad q_{1}=Q_{0}+F V \quad q_{2}=0$ $V=-\frac{1}{F}\left(\sqrt{\frac{A}{K_{1}}}-Q_{0}\right)$

Ensuite $q_{2}$ et $\varepsilon_{2}$ deviennent positifs.

L'intégration graphique s'effectue suivant les mêmes principes que pour la fermeture et permet de déterminer, en particulier, la descente maximum du plan d'eau dans la chambre.

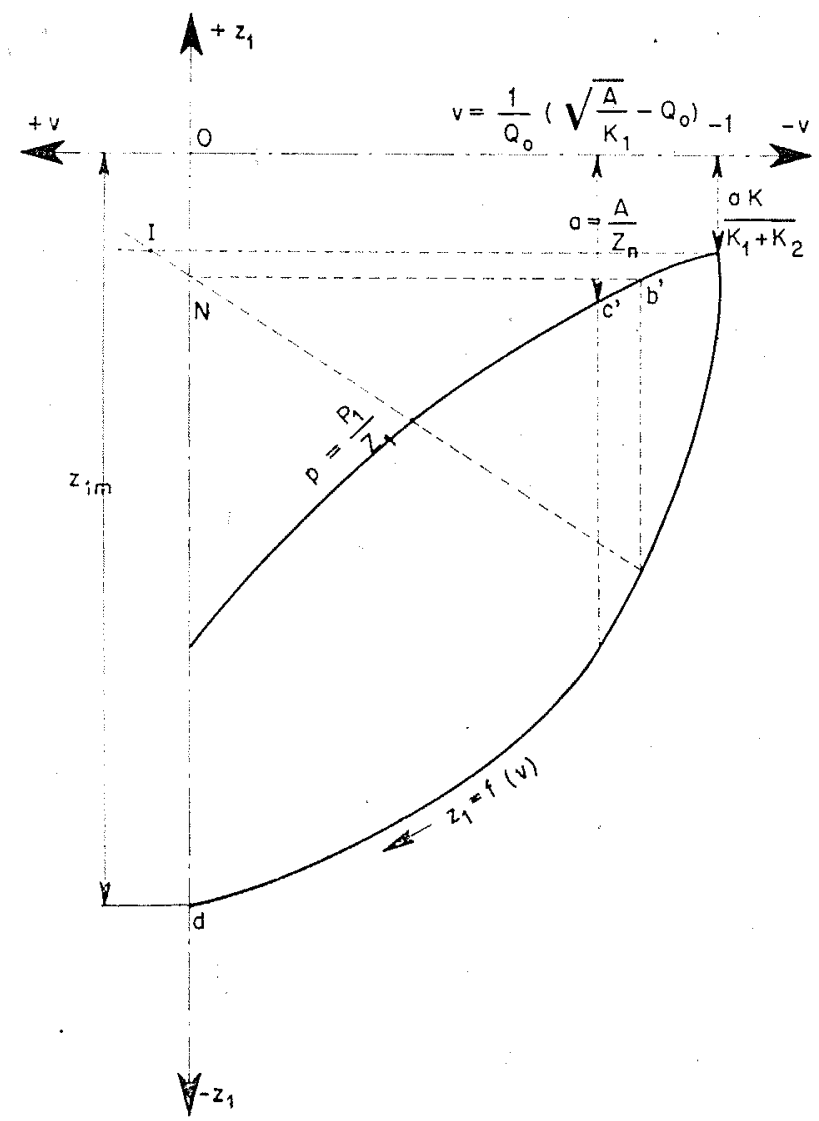

fig. 4
En passant aux valeurs relatives, l'équation de mouvement s'écrit toujours:

$$
\frac{d v}{d z}=-\frac{z_{1}+p_{1}}{v}
$$

mais les conditions initiales sont :

$$
z_{1}=-p_{1} \frac{d v}{d z}=0 \quad v=-1 " \rho_{0}=1
$$

La construction graphique correspondante est indiquée sur la figure 4.

\section{CAS PARTICULIER}

\section{Lacs au même niveau $(A=0)$}

L'expression de la perte de charge devient:

$$
\dot{P}_{1}^{\prime}=P_{2}=\frac{1}{\left(\frac{1}{\left.\sqrt{k_{1}}+\frac{1}{\sqrt{k_{2}}}\right)^{2}}\left(F V+Q_{0}\right)^{2}\right.}
$$

ou bien, fonction de $P_{0}$ :

$$
P_{1}=P_{0}(1+v)^{2}
$$

et en valeurs relatives:

$$
\mathrm{p}_{1}=\mathrm{p}_{0}(1+\mathrm{v})^{2}
$$

L'équation de mouvement devient ainsi :

$$
\frac{d v}{d z}=-\frac{z_{1}+p_{0}(1+v)^{2}}{v}
$$

La méthode s'applique sans difficultés aussi bien au cas des manœuvres partielles et linéaires de l'obturateur, qu'ò l'étude des chambres à section multiples, à étranglement inférieur, etc...

Ainsi dans le cas d'une chambre à étranglement inférieur, lors d'une fermeture totale instantanée, par exemple, il suffirait pendant la $1^{\text {rw }}$ phase de montée, d'intégrer suivant les mêmes principes, l'équation de mouvement :

$$
\frac{d V}{d Z}=-C \frac{Z_{1}+P_{1}+R_{0} v^{2}}{V}
$$


dans laquelle $R_{0}$ représente la perte de charge créée par l'étranglement lorsque celui-ci est traversé par le débit permanent $Q_{0}$ avant fermeture.

En valeurs relatives, l'équation (23) prend la forme ci-après :

$$
\frac{d v}{d z}=-\frac{z_{1}+p_{1}+r_{0} v^{2}}{v}
$$

Lors d'une ouverture, pendant la $1^{\text {to }}$ phase de descente, l'équation de mouvement s'écrirait :

$$
\frac{d v}{d z}=-\frac{z_{1}+p_{1}-r_{0} v^{2}}{v}
$$

En collaboration avec $M$. ESCANDE, nous justifierons, dans un prochain article, I'utilisation pratique de la méthode graphique que nous venons d'exposer, en comparant les résultats obtenus à l'aide de cette dernière et de la méthode aux Différences Finies, appliquées successivement au calcul de la Chambre d'Equilibre complexe de I'Usine de Bioge.

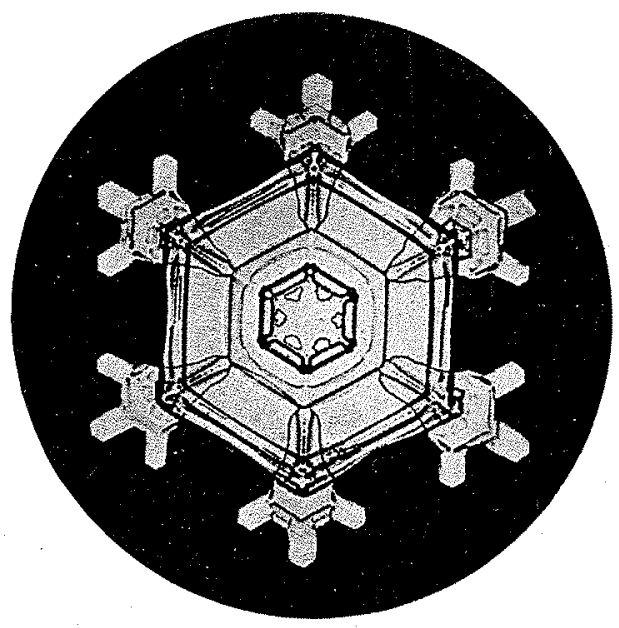

